\title{
Comparison of Stochastic Mortality Model for Wider Age Range
}

\author{
Siti Rohani Mohd Nor, Fadhilah Yusof* and Arifah Bahar \\ Department of Mathematical Sciences \\ Universiti Teknologi Malaysia, Skudai, Johor \\ *Corresponding author: fadhilahy@utm.my
}

Article history

Received: 6 November 2017

Received in revised form: 8 March 2018

Accepted: 3 April 2018

Published on line: 1 December 2018

\begin{abstract}
The incorporation of non-linear pattern of early ages has led to new research directions on improving the existing stochastic mortality model structure. Several authors have outlined the importance of encompassing the full age range in dealing with longevity risk exposure, by not ignoring the dependence between young and old ages. In this study, we consider the two extensions of the Cairns, Blake and Dowd model that incorporate the irregularity profile seen at the mortality of lower ages, which are the Plat, and the O'Hare and Li models respectively. The models' performances in terms of in-sample fitting and out-sample forecasts were examined and compared. The results indicated that the O'Hare and Li model performs better as compared to the Plat model.
\end{abstract}

Keywords Stochastic mortality model; forecasting; non-linearity; analysis of residuals .

Mathematics Subject Classification 91G80

\section{Introduction}

Recent decades uncovered a growing interest in the demographic modelling, in order to cope with the longevity risk that has been rising significantly in most developed countries. Such approaches of this research explosion include: Lee and Carter [1], O'Hare and Li [2], Plat [3], Cairns et al. [4], Renshaw and Haberman [5], and Tuljapurkar [6]. The assessment of the longevity risk is performed by analysing the historical mortality rates across years and across ages. Next, three phases of numerical procedures are implemented, which are historical data fitting, residual measurement error, and mortality projection. Hunt and Blake [7] provided a general guideline to construct a mortality model that could adequately capture all the relevant information related between these three phases. In addition, they listed several desirable characteristics that are required to be met by a good mortality model, which are 1) the model's adequacy is suited to the observed data, 2) the model's parameters are constant with the data changes, 3) the model is biologically reasonable, 4) the model is parsimonious, 5) the model incorporates the cohort parameter if necessary, and 6) the model spans the full age range.

O'Hare and Li [2] are some of the authors that have put emphasis on the importance of including the younger generation below 20's in their modelling procedures. They have expanded 
the Plat [3] and Cairns et al. [4] models into a wider age range. An important aspect to them is that the model must be able to predict similar assumption of mortality improvements over time for all age spans. More than that, information at the early ages is vital to be considered for a model that assimilates the cohort effect. This is because it could give sufficient insight for the more recent birth cohorts' estimates. O'Hare and Li [2] added that the mortality at all given ages are dependent on each other, in which the mortality at the senescence ages are affected by the mortality at the younger ages. Consequently, the average life expectancy is influenced by the mortality changes at all ages. Other than that, an essential amount of early age analyses is important to be incorporated into the model designs since this work as fundamental knowledge for the future analysis of later life (Hauser and Weir [8]; Weir [9]).

Motivated by the importance of the incorporation of all age range in the research designs, this study evaluates and compares two distinct types of stochastic mortality models proposed by Plat [3], and O'Hare and $\mathrm{Li}$ [2] to a Malaysian mortality dataset for the five-year age-groups from 0 to 80, starting from the year 1980 until 2015. To the best of our knowledge, both Plat [3] and O'Hare and Li [2] methods have never been applied to Malaysian mortality data. The models' adequacy for ex-ante and ex-post are assessed by using Mean Absolute Percentage Error (MAPE), Explanation Ratio (ER), and analysis of residuals.

The structure of the paper is organised as follows: Section 2 describes the models' structure of Plat [3], and O'Hare and Li [2]. Section 3 summarises the in-sample fit and out-sample fit performances for both of the applied methods. Finally, Section 4 gives a brief conclusion remark for the paper and for future studies.

\section{Methodology}

In this section, we explore two different types of stochastic mortality models proposed by Plat [3] and O'Hare and Li [2]. However, in this paper we do not include the cohort structure from both methods to ensure flexibility and parsimony of the models (Hahn [10]).

\section{$2.1 \quad$ Plat Model}

Plat [3] expanded the stochastic mortality model from Cairns et al. [4] by allowing the whole age range to be fitted to the model. The equation of the developed model is given by:

$$
\ln \left(m_{x, t}\right)=a_{x}+k_{t}^{1}+k_{t}^{2}(\bar{x}-x)+k_{t}^{3}\left((\bar{x}-x)^{+}\right)+\varepsilon_{x, t}
$$

where

- $m_{x, t}$ is the central mortality rate for age range $x$ in time $t$. The mortality rate is obtained by $m_{x, t}=D_{x, t} / E_{x, t}$ where $D_{x, t}$ is the number of death and $E_{x, t}$ is the number of exposure.

- $a_{x}$ is the age effect parameter defined as $a_{x}=\frac{1}{N} \sum_{t=1}^{N} \ln \left(m_{x, t}\right)$

- $k_{t}^{m}$ where $m=1,2,3$ are the period effect parameters

- $\bar{x}$ is the sample average of the age groups $x$

- $(\bar{x}-x)^{+}=\max (\bar{x}-x, 0)$

- $\varepsilon_{x, t}$ is the error structure. 
Plat [3] and O'Hare and Li [2] adopted the Maximum Likelihood Estimation (MLE) of Poisson distribution for stochastic mortality model's parameter estimation:

$$
D_{x, t}=\operatorname{Poisson}\left(E_{x, t} m_{x, t}\right)
$$

where $D_{x, t}$ is the number of deaths and $E_{x, t}$ is the number of exposure. The log-likelihood function of (2) is maximised as:

$$
L(\emptyset ; D, E)=\sum_{x, t}\left\{D_{x, t} \ln \left[E_{x, t} m_{x, t}(\emptyset)\right]-E_{x, t} m_{x, t}(\emptyset)-\ln \left(D_{x, t} !\right)\right\} .
$$

After the mortality model was applied to the historical dataset, the Box-Jenkins procedure followed, and the period effects were modelled.

\subsection{O'Hare and Li Model}

O'Hare and Li [2] shared similar notations and fitting approach as Plat [3] model. However, they differ in terms of the incorporation of the quadratic coefficient into the model structure. The modified model can be expressed as:

$$
\left.\ln \left(m_{x, t}\right)=a_{x}+k_{t}^{1}+k_{t}^{2}(\bar{x}-x)+k_{t}^{3}\left((\bar{x}-x)^{+}+\left[(\bar{x}-x)^{+}\right]^{2}\right)+\varepsilon_{x, t}\right)
$$

where the non-linear features of $(\bar{x}-x)^{+}+\left[(\bar{x}-x)^{+}\right]^{2}$ are said to capture the irregular characteristics happening during the early mortality experiences.

\subsection{Statistical Measures}

There are several numerical techniques that can be employed in order to evaluate and compare the models' goodness of fit. Some of the proposed methods are:

\subsubsection{Explanation Ratios}

The explanation ratio (ER) is used to measure the variance proportion of the model's residuals. The model has a better fit to the data if the ER value is higher. Meanwhile, the model with the ER measurement below $90 \%$ is considered weak (Enchev et al. [11]).

$$
\mathrm{ER}=1-\frac{\sum_{x, t, i}\left[\log \left(m_{t, x}\right)-\log \left(\hat{m}_{t, x}\right)\right]^{2}}{\sum_{x, t, i}\left[\log \left(m_{t, x}\right)-a(x, i)\right]^{2}}
$$

where $m_{t, x}$ is denoted as the observed mortality rate and $\hat{m}_{t, x}$ is denoted as the predicted mortality rate.

\subsubsection{Standard Residuals}

The Royston test is used to assess the multivariate normality assumption of the models' standardised residuals. The formula for the standardised residuals series is given as below:

$$
S R_{t, x}=\frac{m_{t, x}-\widehat{m}_{t, x}}{\sqrt{\widehat{m}_{t, x} / E_{t, x}}}
$$

where $S R_{t, x}$ is assumed to follow independent and identically distributed normal. 


\section{$3 \quad$ Results and Discussions}

In this section, firstly several quantitative comparisons of Plat and O'Hare and Li models were carried out using Malaysian mortality data for male and female, given the age ranges from 0 to 80, 20 to 80 , and 50 to 80 . The years 1981 to 2010 were selected as an in-sample fitting period, and 2011 to 2015 were used as an out-sample forecast period. There are three steps of model diagnostics presented here for comparison purposes which are: 1) the in-sample fitting, 2) the models' residuals analysis and 3) the out-sample forecasts.

\subsection{The In-Sample Fit Performance}

Table 1 and Table 2 lists the goodness of fit results of MAPE and ER for both of the corresponding models in the percentage form (\%). The best performing model is determined by the lowest MAPE and highest ER values, which are indicated by bolded text. Different age ranges were fitted to both the Plat and O'Hare and Li model, to compare the performances of the two methods when the quadratic mortality effects are incorporated into the modelling structure. More than that, the models were applied to the datasets of male and female to see the significant differences of the models performance when fitted to the apparent and non-apparent non-linear mortality pattern displayed for the lower ages of male and female.

Table 1: The In-sample MAPE for Plat and O'Hare and Li Models (\%)

\begin{tabular}{|l|c|c|}
\hline Model & Plat & O'Hare \\
\hline \multicolumn{2}{|l|}{ Ages 0 to 80 (\%) } & 6.318 \\
\hline Male & 8.013 & 5.070 \\
\hline Female & 5.247 & 5.285 \\
\hline Ages 20 to 80 (\%) & 4.138 \\
\hline Male & 5.336 & 2.829 \\
\hline Female & 4.219 & 3.611 \\
\hline Ages 50 to 80 (\%) & 2.817 & \\
\hline Male & 3.461 & \\
\hline Female
\end{tabular}

According to Table 1, the O'Hare and Li model outperformed the Plat model when the age ranges are widened to the early mortality experiences, which are from 0 to 80, and from 20 to 80. In addition, this result indicated that the O'Hare and Li method could give better fit to the apparent and non-apparent non-linear pattern of the male and female at the early ages. However, when both of the stochastic mortality models were fitted to the advanced ages of 50 to 80, the Plat model performed better as compared to O'Hare and Li method with a slight change of $0.1 \%$. Then, the explanation ratios in equation (4) were applied to the mortality residuals. Similar to Table 1, the results of ER in Table 2 reveal that the O'Hare and Li model 
Table 2: The Explanation Ratios for Plat and O'Hare and Li Models (\%)

\begin{tabular}{|l|l|l|l|}
\hline Model & \multicolumn{3}{|c|}{ Explanation Ratio (\%) } \\
\hline Age Groups & 0 to 80 & 20 to 80 & 50 to 80 \\
\hline Plat & 80.07 & 80.56 & 87.09 \\
\hline O'Hare and Li & 85.46 & 80.86 & 86.66 \\
\hline
\end{tabular}

performs the best when fitted to the wider age range, but has a poor fit performance when fitted to the later life.

Next, the residuals obtained from the in-sample fit performances were analysed using multivariate normality distribution test which is the Royston test. The residuals analysis outcomes are tabulated in Table 3.

Table 3: Residuals Analysis for Plat and O'Hare and Li Models

\begin{tabular}{|l|l|l|l|l|}
\hline & \multicolumn{2}{|c|}{ Male } & \multicolumn{2}{c|}{ Female } \\
\hline & Plat & O'Hare and Li & Plat & O'Hare and Li \\
\hline Mean & 0.165 & 0.085 & 0.127 & 0.121 \\
\hline Variance & 25.379 & 16.707 & 12.114 & 11.495 \\
\hline Skewness & 0.288 & 0.068 & 0.270 & 0.225 \\
\hline Kurtosis & 2.831 & 3.163 & 4.607 & 4.829 \\
\hline Mean & 0.448 & 0.630 & 0.397 & 0.408 \\
\hline Royston & 0.015 & 0.019 & 0.020 & 0.016 \\
\hline
\end{tabular}

The results from Table 3 shows that the residuals of Plat and O'Hare and Li methods for both male and female do not follow multivariate normality assumptions, since the null hypothesis of Royston test is rejected. O'Hare and Li [12] and Dowd et al. [13] have obtained similar results in which all the leading stochastic models that they considered failed to follow the normality tests assumption. O'Hare and $\mathrm{Li}[12]$ stated that the non normality residuals are a small issue for comparison mortality model purposes, however it is a significant issue that needs to be considered for the actuarial application purposes. Hence, one of the methods that could be used to overcome this problem is by repeatedly doing the refitting and forecasting process.

\subsubsection{The Out-Sample Forecast Performance}

These sub-sections discuss the out-sample fit performances of male and female projected for a 5-year period starting from the year 2011 until 2015. We empirically compared the O'Hare-Li and Plat models for the three distinct age ranges which are 0 to 80, 20 to 80 and 50 to 80 . 
Table 4 compares the error measures based on the mortality projection for Plat and O'HareLi methods by using MAPE. The empirical results are not consistent with the results reported in Table 1 for the group of ages 0 to 80, and 50 to 80. It can be seen that the Plat model outperformed the O'Hare and Li model for male at the age 0 to 80, and for female at the age 50 to 80. On the other hand, the O'Hare and Li model gives the best projection accuracy for female at the age 0 to 80 , for both male and female at the age 20 to 80 , and for male at the age 50 to 80 .

Table 4: The Out-sample MAPE for Plat and O'Hare and Li models (\%)

\begin{tabular}{|l|c|c|}
\hline Model & Plat & O'Hare \\
\hline Ages 0 to 80 (\%) & 8.489 & 9.731 \\
\hline Male & 9.097 & 8.067 \\
\hline Female & 9.500 \\
\hline Ages 20 to 80 (\%) & 11.020 & 8.817 \\
\hline Male & 8.913 & 2.808 \\
\hline Female & 3.403 & 7.734 \\
\hline Ages 50 to 80 (\%) & 5.517 & \\
\hline Fale &
\end{tabular}

\section{Conclusion}

In this study, evaluations and comparisons were made for the two existing stochastic mortality models proposed by Plat and O'Hare and Li applied on the Malaysian mortality data for the full age span's purposes. As opposed to Plat, O'Hare and Li method differs in the quadratic effect age parameter in which it allows the nonlinear mortality behaviour occurring at the lower ages below 20's to be incorporated into the model. According to the Mean Absolute Percentage Error and Explanation Ratios measurements, the O'Hare and Li method outperformed the Plat model in terms of the in-sample fit performances at the lower ages. This means the O'Hare and Li model could more accurately capture the dynamics of the young mortality experience below 20's as compared to Plat. More than that, O'Hare and Li have a better out-sample forecast performances for female at the age of 0 to 80 , and both male and female at the age of 20 to 80. The out-sample forecast results are not consistent with the results obtained in the in-sample fit, which might be due to the resulting residuals of both methods that fail to follow the multivariate normality assumptions. This indicates that both the Plat and O'Hare and Li models need to be modified and improved so that it could be applicable for Malaysian mortality data for forecasting purposes. The results that we have obtained in this study will be used as the preliminary study on the multi-population mortality model for Malaysian data. 


\section{Acknowledgements}

The authors would like to thank Department of Statistics Malaysia (DOSM) for providing the data and we would like to acknowledge Universiti Teknologi Malaysia (UTM) andMinistry of High Education (MOHE) for the financial resources under the Skim Latihan Anak Muda (SLAM) scholarship and research STEM grant with vote no. A. J091002.5600.07397.

\section{References}

[1] Lee, R. D. and Carter, L. R. Modeling and Forecasting U. S. Mortality. Journal of the American Statistical Association.1992. 87(419): 659-671.

[2] O'Hare, C. and Li, Y. Explaining young mortality. Insurance: Mathematics and Economics. 2012. 50(1): 12-25.

[3] Plat, R. On stochastic mortality modeling. Insurance: Mathematics and Economics. 2009. 45(3): 393-404.

[4] Cairns, A. J. G., Blake, D., Dowd, K., Coughlan, G. D., Epstein, D., Ong, A. and Balevich, I. A quantitative comparison of stochastic mortality models using data from England and Wales and the United States. North American Actuarial Journal. 2009. 13: 1-35.

[5] Renshaw, A. E. and Haberman, S. A cohort-based extension to the Lee-Carter model for mortality reduction factors. Insurance: Mathematics and Economics. 2006. 38(3): 556-570.

[6] Tuljapurkar, S. Mortality declines, longevity risk and aging. Asia-Pacific Journal of Risk and Insurance. 2008. 3(1): 37-51.

[7] Hunt, A. and Blake, D. A General Procedure for Constructing Mortality Models. North American Actuarial Journal. 2014. 18(1): 116-138.

[8] Hauser, R. M. and Weir, D. Recent developments in longitudinal studies of aging. Demography. 2016. 23(5): 1079-1084.

[9] Weir, D. R. Grand challenges for the scientific study of ageing. American Economic Association, Ten Years and Beyond: Economists Answer NSF's Call for Long-Term Research Agendas. Available at SSRN: https://ssrn.com/abstract=1889373. 2010.

[10] Hahn, L. J. A Bayesian Multi-Population Mortality Projection Model. University of Ulm. Master's Thesis. 2014.

[11] Enchev, V., Kleinow, T. and Cairns, A. J. G. Multi-population mortality models: fitting, forecasting and comparisons. Scandinavian Actuarial Journal. 2016.4: 319-342.

[12] O'Hare, C. and Li, Y. Models of mortality rates - analysing the residuals. Applied Economics. 2017. 49(52): 5309 - 5323

[13] Dowd, K., Cairns, A. J. G., Blake, D., Coughlan, G. D., Epstein, D. and Khalaf-Allah, M. Evaluating the goodness of fit of stochastic mortality models. Insurance: Mathematics and Economics. 2010. 47(3): 255-265. 